\title{
CovidCTNet: an open-source deep learning approach to diagnose covid-19 using small cohort of CT images
}

\author{
Tahereh Javaheri ${ }^{1,22}$, Morteza Homayounfar ${ }^{2,22}$, Zohreh Amoozgar ${ }^{3,22}$, Reza Reiazi iD ${ }^{4,5,6,22}$, Fatemeh Homayounieh ${ }^{7}$, Engy Abbas ${ }^{8}$, \\ Azadeh Laali ${ }^{9}$, Amir Reza Radmard (iD ${ }^{10}$, Mohammad Hadi Gharib ${ }^{11}$, Seyed Ali Javad Mousavi ${ }^{12}$, Omid Ghaemi ${ }^{10}$, Rosa Babaei ${ }^{13}$, \\ Hadi Karimi Mobin ${ }^{13}$, Mehdi Hosseinzadeh ${ }^{14,15}$, Rana Jahanban-Esfahlan ${ }^{16}$, Khaled Seidi ${ }^{16}$, Mannudeep K. Kalra (iD) \\ Guanglan Zhang ${ }^{1,17}$, L. T. Chitkushev ${ }^{1,17}$, Benjamin Haibe-Kains ${ }^{4,5,18,19,20}$, Reza Malekzadeh ${ }^{21}$ and Reza Rawassizadeh (iD) ${ }^{1,17 凶}$
}

Coronavirus disease 2019 (Covid-19) is highly contagious with limited treatment options. Early and accurate diagnosis of Covid-19 is crucial in reducing the spread of the disease and its accompanied mortality. Currently, detection by reverse transcriptasepolymerase chain reaction (RT-PCR) is the gold standard of outpatient and inpatient detection of Covid-19. RT-PCR is a rapid method; however, its accuracy in detection is only $~ 70-75 \%$. Another approved strategy is computed tomography (CT) imaging. CT imaging has a much higher sensitivity of $\sim 80-98 \%$, but similar accuracy of $70 \%$. To enhance the accuracy of CT imaging detection, we developed an open-source framework, CovidCTNet, composed of a set of deep learning algorithms that accurately differentiates Covid-19 from community-acquired pneumonia (CAP) and other lung diseases. CovidCTNet increases the accuracy of CT imaging detection to $95 \%$ compared to radiologists (70\%). CovidCTNet is designed to work with heterogeneous and small sample sizes independent of the CT imaging hardware. To facilitate the detection of Covid-19 globally and assist radiologists and physicians in the screening process, we are releasing all algorithms and model parameter details as open-source. Open-source sharing of CovidCTNet enables developers to rapidly improve and optimize services while preserving user privacy and data ownership.

npj Digital Medicine (2021)4:29; https://doi.org/10.1038/s41746-021-00399-3

\section{INTRODUCTION}

In the era of communication, the current epidemic of highly contagious Covid-19 (SARS-Cov-2) has negatively impacted the global health, trade, and economy. To date, the mortality rate of Covid-19 is estimated to be 35-45 times higher than the pandemic influenza, accounting for more than $1,000,000$ deaths ${ }^{1-4}$. Covid-19 has surpassed its predecessors SARS-CoV, and MERS-CoV, in morbidity and mortality ${ }^{5}$. Unfortunately, the longterm studies on SARS-CoV, the cause of SARS $^{6}$, did not find effective and safe treatments ${ }^{7}$. Lack of effective therapy underlines the importance of early diagnosis, rapid isolation, and strict infection control to minimize the spread of Covid-19.

Currently, diagnosis is mainly based on the patient's medical history, RT-PCR, and CT imaging ${ }^{8-12}$. High error (30-35\%) of RT$P C R^{8,9,13}$, lack of distinction between viral contamination versus disease-bearing individuals ${ }^{14}$ or false-positive/negative ${ }^{15}$ may have contributed to the high prevalence of Covid-19 and the dismal therapeutic outcomes. Here, CT imaging plays a critical role in Covid-19 diagnosis since it not only detects the presence of disease in the lung but also enables identifying the stage of the disease by scoring the $\mathrm{CT}$ images ${ }^{9,16,17}$. CT imaging, however, has its own limitations that need to be addressed. The lack of specificity and the similarities between the lung lesions generated by other types of viral infection or community-acquired pneumonia (CAP) may contribute to misdiagnosis for Covid-19 ${ }^{18-20}$. We hypothesized that using robust tools such as machine learning can resolve the $\mathrm{CT}$ imaging technical bias and corrects for human errors ${ }^{17,21-26}$.

An appropriate machine learning framework for Covid-19 detection should (i) be able to assist radiologists and their staff to rapidly and accurately detect Covid-19, (ii) be compatible with a wide range of image scanning hardware's, and (iii) be user friendly to the medical community without computer-science expertize. In our effort to address the clinical diagnostic needs in the Covid-19 pandemic crisis under institutional review board (IRB) approval (IR. TUMS.VCR.REC.1399.007), we designed CovidCTNet framework. CovidCTNet is composed of a pipeline of deep learning algorithms trained on identifying Covid-19 lesions in lung CT images to improve the process of Covid-19 detection.

While deep learning approaches used for Covid-19 detection require large datasets ${ }^{27-29}$, CovidCTNet by employing BCDU-Net ${ }^{30}$ requires only a small sample size for training to achieve accurate detection of Covid-19 without potential bias. For these reasons, our model is significantly different from other models ${ }^{25,29,31,32}$

\footnotetext{
${ }^{1}$ Health Informatics Lab, Metropolitan College, Boston University, Boston, USA. ${ }^{2}$ Department of Biomedical Engineering, Amirkabir University of Technology, Tehran, Iran. ${ }^{3}$ Department of Radiation Oncology, Massachusetts General Hospital, Harvard Medical School, Boston, USA. ${ }^{4}$ Princess Margaret Cancer Centre, University of Toronto, Toronto, Canada. ${ }^{5}$ Department of Medical Biophysics, University of Toronto, Toronto, Canada. ${ }^{6}$ Department of Medical Physics, School of Medicine, Iran university of Medical Sciences, Tehran, Iran. ${ }^{7}$ Department of Radiology, Massachusetts General Hospital, Harvard Medical School, Boston, USA. ${ }^{8}$ Joint Department of Medical Imaging, University of Toronto, Toronto, Canada. ${ }^{9}$ Department of Infectious Diseases, Firoozgar Hospital, Iran University of Medical Sciences, Tehran, Iran. ${ }^{10}$ Department of Radiology, Shariati Hospital, Tehran University of Medical Sciences, Tehran, Iran. ${ }^{11}$ Department of Radiology and Golestan Rheumatology Research Center, Golestan University of Medical Sciences, Gorgan, Iran. ${ }^{12}$ Department of Internal Medicine, Iran University of Medical Sciences, Tehran, Iran. ${ }^{13}$ Department of Radiology, Iran University of Medical Sciences, Tehran, Iran. ${ }^{14}$ Institute of Research and Development, Duy Tan University, Da Nang, Vietnam. ${ }^{15} \mathrm{Health}$ Management and Economics Research Center, Iran University of Medical Sciences, Tehran, Iran. ${ }^{16}$ Department of Medical Biotechnology, School of Advanced Medical Sciences, Tabriz University of Medical Sciences, Tabriz, Iran. ${ }^{17}$ Department of Computer Science, Metropolitan College, Boston University, Boston, USA. ${ }^{18}$ Department of Computer Science, University of Toronto, Toronto, ON, Canada. ${ }^{19}$ Ontario Institute for Cancer Research, Toronto, ON, Canada. ${ }^{20}$ Vector Institute for Artificial Intelligence, Toronto, ON, Canada. ${ }^{21}$ Digestive Disease Research Center, Tehran University of Medical Sciences, Tehran, Iran. ${ }^{22}$ These authors contributed equally: Tahereh Javaheri, Morteza Homayounfar, Zohreh Amoozgar, Reza Reiazi. ${ }^{凶}$ email: rezar@bu.edu
} 
which requires a large dataset of CT images. In our framework, we first applied multiple pre-processing steps on CT images using BCDU-Net ${ }^{30}$ which is designed based on the U-Net ${ }^{33,34}$, a wellknown convolutional network for biomedical image analysis. BCDU-Net is an optimum network due to memory (LSTM cells), allowing the model to remember the structure of the healthy lung. In particular, the CovidCTNet used BCDU-Net to (i) clean images, i.e., removing the image segments unrelated to infection, such as heart, skin, or the bed of CT image device and (ii) train a noise cancellation model, which was used by our model to extract infection. Note that both Covid-19 and CAP are associated with a lung infection, and visually they are very similar. Therefore, a robust Covid-19 identification approach should distinguish them accurately. Otherwise, classification algorithms cannot distinguish Covid-19, CAP, and control lungs in the small dataset and from original CT images. However, with the assistance of BCDU-Net, our model cleaned the $\mathrm{CT}$ images from other tissues, except the lung infection.

After the process of infection extraction, the result of CT images was fed into a convolutional neural network (CNN) to classify the given CT images as control, CAP, or Covid-19. All of our codes, including details of model parameters, are clearly explained and released as open-source. In this study, we developed the CovidCTNet, which consists of a pipeline of deep learning algorithms to accurately detect Covid-19 infection. A heterogeneous dataset was analyzed in this study to ensure that CovidCTNet can address the needs of hospitals across the globe, irrespective of the sample size, an imaging device (hardware), or the imaging software.

\section{RESULTS}

\section{Extraction of Covid-19 lesions from CT images}

We assessed a dataset consisting of 16,750 slices of all CT scan images from 335 patients. Among this dataset, 111 (5550 CT slices) patients were infected with Covid-19 with a confirmed RT-PCR, patient's medical history, and radiologist diagnosis. The second cohort was 115 (5750 CT slices) patients infected with CAP or other viral sources with $\mathrm{CT}$ images that can be potentially misdiagnosed for Covid-19. Our Control group consists of a cohort of patients 109 (5450 CT slices) with healthy lungs or other non-Covid-19/non-CAP diseases. Additionally, a cohort of 70 CT scans was used from SPIE-AAPM-NCI lung nodule classification challenge dataset ${ }^{35}$, a heterogeneous dataset that contains lung cancer as well (summarized in Tables 1 and 2). 66 cases $(21,888$ CT slices) out of 70 were randomly selected for training and validation phases. Four cases served as a control for reader tests. CT images were acquired from multiple institutions, including five medical centers in Iran, a country that is highly affiliated with Covid-19 and from publicly available dataset from lung nodule classification (LUNGx) challenge, an archive generated by the University of Chicago $^{35,36}$.

The dataset was collected from 12 different CT scanner models of five different brands. Our sample size was small and to achieve a high performing model that is operational and unbiased, we used BCDU-Net as the backbone of our model. To identify Covid19 in the lung as well as CAP lesion, we generated pseudoinfection anomalies in the CT control images using Perlin noise ${ }^{37}$.

\section{CovidCTNet mitigates the challenge of small dataset by highlighting the infection}

To test whether applying Perlin noise and using BCDU-Net is necessary for preprocessing and if they increase the accuracy of our model, we conducted a validation experiment. The 3D CNN model was performed with and without the use of BCDU-Net and Perlin noise. The implementation of BCDU-Net significantly boosted the accuracy of the model and demonstrated the importance of using Perlin noise and preprocessing steps. Figure 1 presents binary cross entropy (loss) and accuracy of the CovidCTNet in different conditions. While the accuracy of the model without using BCDU-Net and Perlin noise at the training phase is very high, it drops significantly in the validation phase. This confirms that the features and parameters that were selected by the CNN model (without BCDU-Net) were not sufficient. In addition, applying them significantly changes the accuracy of training and validation, which demonstrates the need for preprocessing in increasing the model robustness. Note that the results shown in Fig. $1 \mathrm{a}$, b were generated by training the model

\begin{tabular}{|c|c|c|c|c|c|}
\hline \#Patient cohort & $\begin{array}{l}\text { CT slices with } \\
\text { Perlin noise }\end{array}$ & $\begin{array}{l}\text { CT slices without } \\
\text { Perlin noise }\end{array}$ & Patients & $\overline{C T}$ slices & $\begin{array}{l}\text { Loss (binary cross-entropy) Optimizer }=\text { Adam } \\
\text { learning rate }=0.001\end{array}$ \\
\hline Preprocessing-train & 9913 & 9914 & 60 & 19,827 & 0.3585 \\
\hline Preprocessing-validation & 1031 & 1030 & 6 & 2061 & 0.3638 \\
\hline
\end{tabular}

Details of individual samples and total cases that were used in preprocessing.

Table 2. Detail information of the samples in multiple steps of analysis (preprocessing, train, validation, and test phases).

\begin{tabular}{|c|c|c|c|c|c|c|c|c|}
\hline \#Patient cohort & \multicolumn{2}{|l|}{ Control } & \multicolumn{2}{|l|}{ CAP } & \multicolumn{2}{|l|}{ Covid-19 } & \multicolumn{2}{|l|}{ Total } \\
\hline Validation & 5 & $5 \times 50$ & 5 & $5 \times 50$ & 5 & $5 \times 50$ & 15 & 750 \\
\hline Reader test & 4 & $4 \times 50$ & 10 & $10 \times 50$ & 6 & $6 \times 50$ & 20 & 1000 \\
\hline Total & 109 & $109 \times 50$ & 115 & $115 \times 50$ & 111 & $111 \times 50$ & 335 & 16,750 \\
\hline
\end{tabular}

A summary of individual samples and total cases that were used in the train, validation, and test phases. To maintain the balance in the dataset, a total number of 100 cases were used for each of Control, CAP, and Covid-19 groups. 
(a)

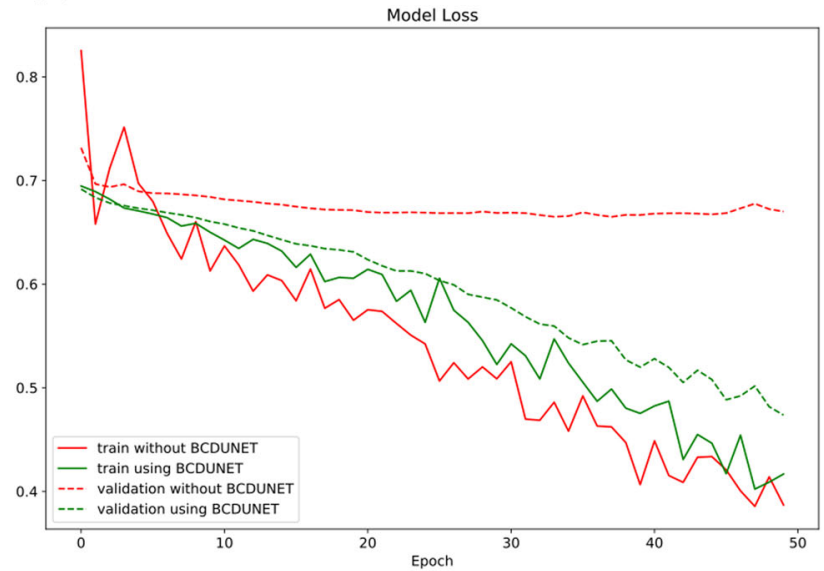

(b)

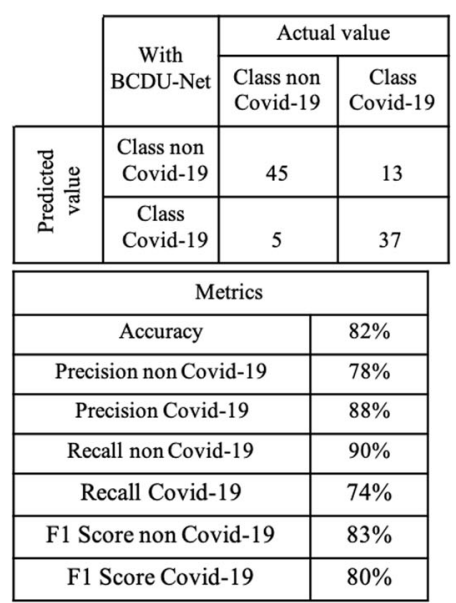

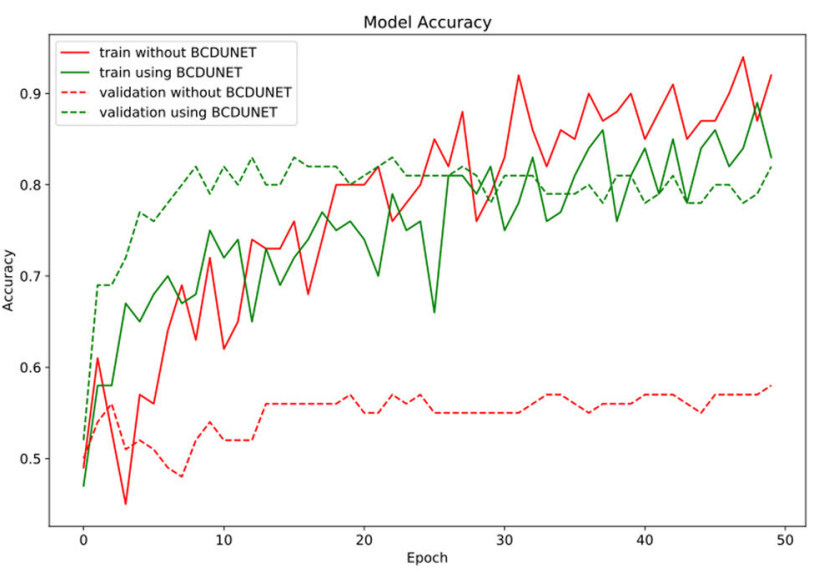

\begin{tabular}{|c|c|c|c|}
\hline & \multirow{2}{*}{$\begin{array}{c}\text { Without } \\
\text { BCDU-Net }\end{array}$} & \multicolumn{2}{|c|}{ Actual value } \\
\hline & & $\begin{array}{l}\text { Class non } \\
\text { Covid-19 }\end{array}$ & $\begin{array}{c}\text { Class } \\
\text { Covid-19 }\end{array}$ \\
\hline \multirow{2}{*}{ 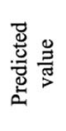 } & $\begin{array}{l}\text { Class non } \\
\text { Covid-19 }\end{array}$ & 29 & 21 \\
\hline & $\begin{array}{c}\text { Class } \\
\text { Covid-19 }\end{array}$ & 21 & 29 \\
\hline \multicolumn{4}{|c|}{ Metrics } \\
\hline \multicolumn{3}{|c|}{ Accuracy } & $58 \%$ \\
\hline \multicolumn{3}{|c|}{ Precision non Covid-19 } & $58 \%$ \\
\hline \multicolumn{3}{|c|}{ Precision Covid-19 } & $58 \%$ \\
\hline \multicolumn{3}{|c|}{ Recall non Covid-19 } & $58 \%$ \\
\hline \multicolumn{3}{|c|}{ Recall Covid-19 } & $58 \%$ \\
\hline \multicolumn{3}{|c|}{ F1 Score non Covid-19 } & $58 \%$ \\
\hline \multicolumn{3}{|c|}{ F1 Score Covid-19 } & $58 \%$ \\
\hline
\end{tabular}

Fig. 1 BCDU-Net increases the robustness of the CNN model. a To show the effect of BCDU-Net on the preprocessing, the procedure was done with and without applying BCDU-Net/Perlin noise. The outcome of the model is presented with respect to loss and accuracy. $\mathbf{b}$ The confusion matrix and other classification related metrics in detail. The results shown in this figure are based on just 50 randomly selected cases for each class of Covid versus non-Covid.

with only 50 cases for each class, which proves the necessity and usefulness of applying the BCDU-Net for a limited amount of data. Figure 2 presents the extracted infection by BCDU-Net in 2D and Fig. 3 presents the extracted infection by BCDU-Net in 3D. The output of BCDU-Net (Fig. 3) will be fed to the CNN model as an input. It can be seen from Figs. 2 and 3 how BCDU-Net reduces the noninfectious parts of the $C T$ image and highlights the infections inside the lung.

\section{CovidCTNet accurately detects Covid-19 from other lung diseases}

The output of the algorithm (a tensor such as the right side of Fig. 3b) was fed into the CNN classification algorithm. In CNN assessment, the dataset was split in $95 \%$ to train the algorithm, and $5 \%$ to validate the model in the hold-out. The area under receiver operating characteristics (ROC) curve (AUC) for Covid-19 at the validation phase was $94 \%$, with an accuracy of $93.33 \%$ when CNN classified Covid-19 versus non-Covid-19 (two classes) (Fig. 4). CNN achieved the accuracy of $86.66 \%$ when it classified Covid-19 versus CAP and Control (three classes). The detection sensitivity of $90.91 \%$ and specificity of $100 \%$ were recorded for Covid-19 (Fig. 4).

\section{CovidCTNet outperforms radiologists}

To test the classification quality of our framework, an independent dataset consisting of 20 cases mixed of Control, Covid-19, and CAP were assessed using our framework and in parallel four certified and independent radiologists who were not involved in the process of data collection. The average reader performance of four radiologists showed a sensitivity of $79 \%$ for Covid-19 and specificity of $82.14 \%$. The CNN classification of CovidCTNet, however outperformed the radiologists and achieved Covid-19 detection with sensitivity and specificity of 93 and 100\%, respectively. Table 3 . details the comparison of radiologist performance versus CovidCTNet.

Radiologists performance accuracy was $81 \%$, while CovidCTNet classification achieved a $95 \%$ accuracy when the question was detecting between Covid-19 versus non-Covid-19 (2 classes). When we asked to detect Covid-19 versus CAP versus control (three classes), again our approach outperformed the radiologists with an accuracy of $85 \%$ compared with human accuracy of $71 \%$. The AUC of the model in Covid-19 detection versus reader test was $90 \%$ (Fig. 5). The accuracy, sensitivity, and specificity of the model showed a significantly higher validity compared to the average of radiologists. 

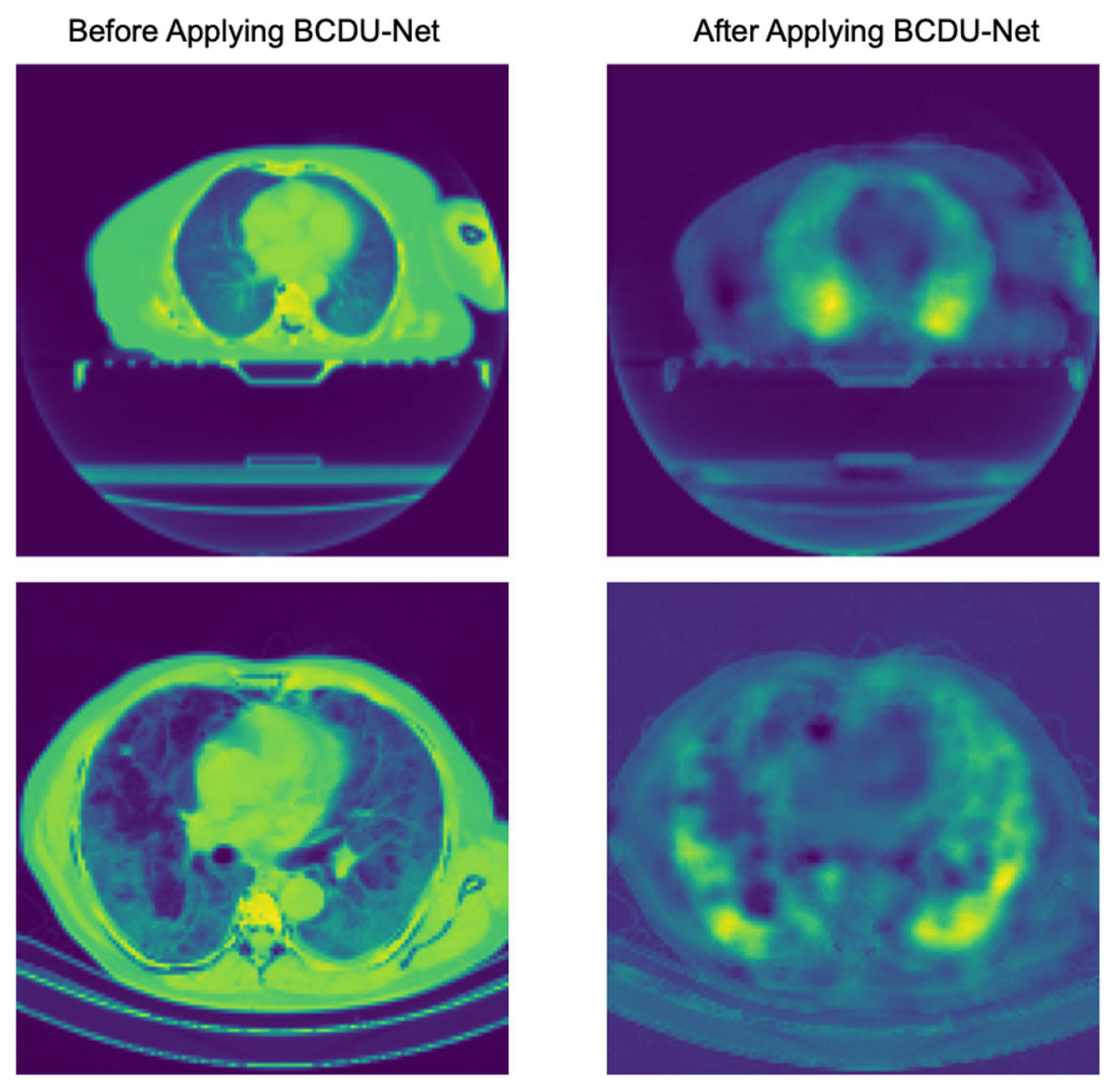

Fig. 2 Covid-19 and CAP infection extraction by BCDU-Net. The filtered images (left) will be used for classification by CNN. An unprocessed 3D image of the whole lung infected with Covid-19 is shown in Fig. 3a. The same image was processed with BCDU-Net to remove non lungrelated parts and to extract and highlight the Covid-19 infection (Fig. 3b).

(a)

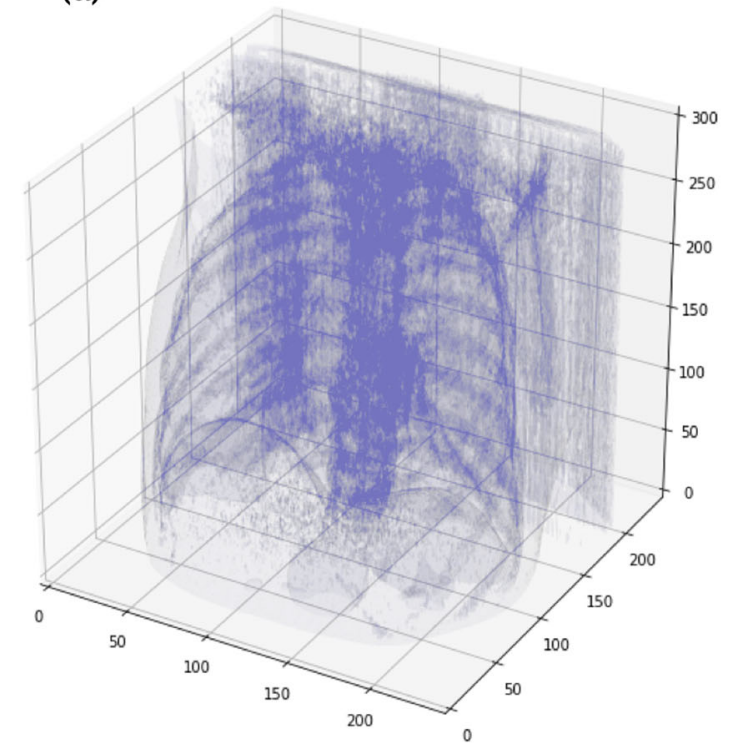

(b)

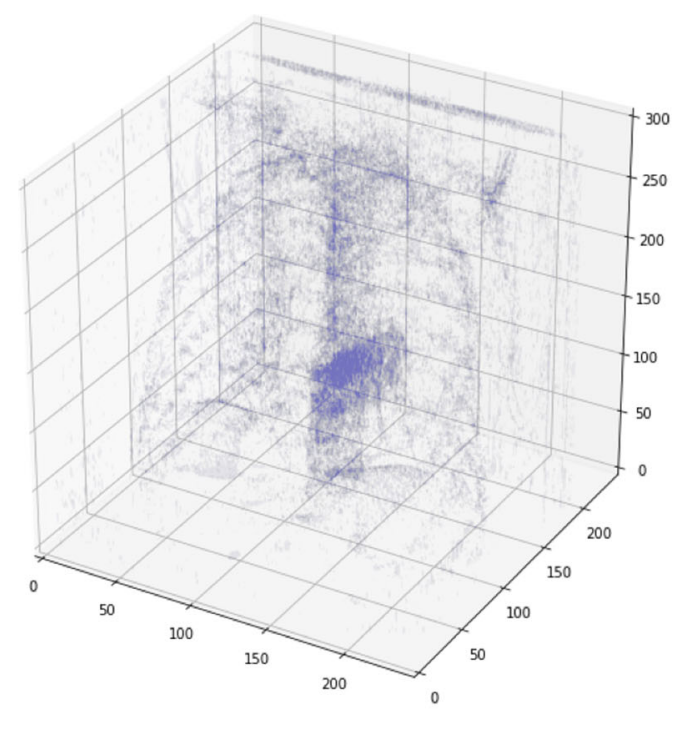

Fig. 3 Schematic representation of BCDU-Net module to detect the infection in CT images. a The original CT images visualized in point cloud. b Reconstructed lung image acquired by feeding the CT slices (Fig. 8 middle part $h$ ) into BCDU-Net. The Covid-19 infection area is highlighted in b.

\section{Images of Covid-19 and CAP infection share structural similarities}

Despite some clear differences in Covid-19 and CAP infection pattern, the high similarities in ground-glass opacities (GGO) and consolidation on chest CT of Covid-19 and CAP (Fig. 4) makes differential detection a challenge. Several suspicious and challenging images are shown in Fig. 6.

The CAP CT slide (Fig. 6a) was misdiagnosed by radiologists as Covid-19 or Control by radiologists but correctly by CovidCTNet. Figure $6 \mathrm{~b}$ is a control image that was correctly 


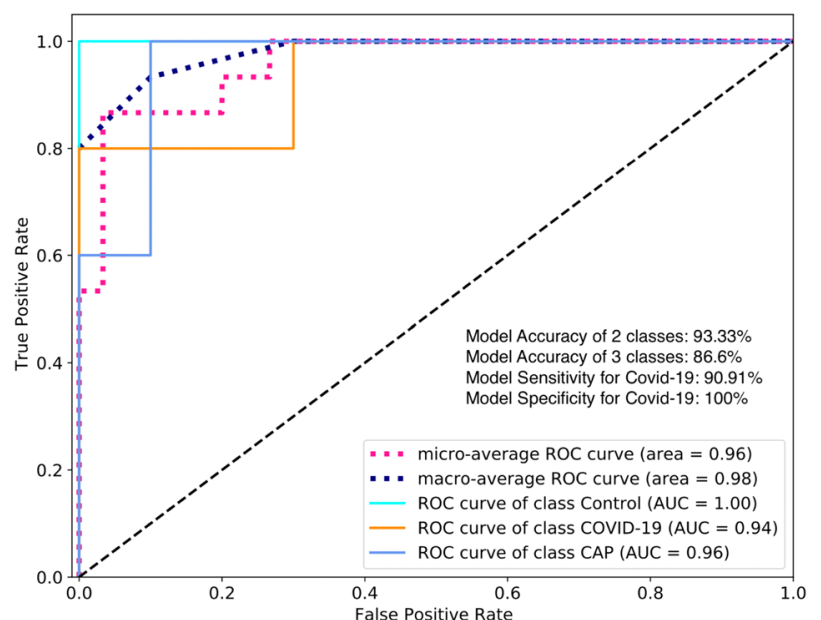

Fig. 4 Performance of CovidCTNet in detecting Control, Covid-19, and CAP. The model's AUC for Covid-19 detection is $0.94(n=15$ cases). The accuracy, sensitivity, and specificity of the model are shown. The model operation in three classes demonstrates the detection of all three classes including Covid-19 versus CAP and versus Control and in two classes indicates the detection of Covid19 as one class versus non-Covid-19 (CAP and Control) as second class.

diagnosed by CovidCTNet and misdiagnosed by three radiologists. Figure $6 \mathrm{c}$ is a Covid-19. Its diagnosis posed a challenge for three radiologists out of four and also for CovidCTNet. The Control shown in Fig. $6 \mathrm{~d}$ was misdiagnosed by two radiologists and by CovidCTNet.

\section{DISCUSSION}

In recent studies the average sensitivity of radiologists to detect the Covid-19 infection is reported to be approximately $70 \%{ }^{20}$, indicating the need for decision support tools to assist radiologists in detecting Covid-19, especially in regions where there is limited in the number of trained clinical staff or the comprehensive expertize to detect Covid-19.

Due to the high value of patch-based classification ${ }^{38}$ we implemented it as one of our models, which did not perform as good as CovidCTNet on the small dataset. The CovidCTNet improved the accuracy and consistency of lung screening for Covid-19 detection through a ready-to-use platform with a sensitivity of $93 \%$ and accuracy of $95 \%$ (in making a binary decision, i.e., Covid-19 and non-Covid19). While the broad similarity of patterns and image features of Covid-19 and CAP posed a challenge for algorithm training, the high accuracy of the model indicates the potential for CovidCTNet to be further refined and adapted as a clinical decision support tool. In contrast to state-of-the-art works ${ }^{17,25,26,29,31,32}$ the dataset we used in this study is significantly smaller and highly heterogeneous. Adding more CT images will increase the accuracy and performance of the model.

Beyond optimizing and improving the Covid-19 detection, CovidCTNet has the potential to significantly impact the clinical workflow and patient care by offering a rapid, inexpensive, and accurate methodology to empower healthcare workers during the pandemic. Our radiologists are highly experienced from prestigious institutions. In an underrepresented region, it is not easy to find an experienced radiologist and we believe these types of $\mathrm{Al}$ systems will be significantly helpful to save lives. Importantly, when an infection type is hard to be diagnosed by the human eye, and when a consensus among radiologists cannot be made,
Table 3. Comparison of the accuracy of CovidCTNet versus radiologists.

\begin{tabular}{|c|c|c|c|}
\hline & Precision & Recall & F1-score \\
\hline \multicolumn{4}{|l|}{ Radiologist 1} \\
\hline Control & 1 & 0.5 & 0.533 \\
\hline CAP & 0.8 & 0.4 & 0.533 \\
\hline Covid-19 & 0.385 & 0.833 & 0.526 \\
\hline Accuracy & 0.55 & & \\
\hline \multicolumn{4}{|l|}{ Radiologist 2} \\
\hline Control & 0.5 & 0.5 & 0.5 \\
\hline CAP & 0.889 & 0.8 & 0.842 \\
\hline Covid-19 & 0.714 & 0.833 & 0.769 \\
\hline Accuracy & 0.75 & & \\
\hline \multicolumn{4}{|l|}{ Radiologist 3} \\
\hline Control & 1 & 1 & 1 \\
\hline CAP & 1 & 1 & 1 \\
\hline Covid-19 & 1 & 1 & 1 \\
\hline Accuracy & 1 & & \\
\hline \multicolumn{4}{|l|}{ Radiologist 4} \\
\hline Control & 0.25 & 0.5 & 0.33 \\
\hline CAP & 0.66 & 0.6 & 0.63 \\
\hline Covid-19 & 1 & 0.5 & 0.66 \\
\hline Accuracy & 0.55 & & \\
\hline \multicolumn{4}{|c|}{ All radiologist average } \\
\hline Control & 0.68 & 0.625 & 0.624 \\
\hline CAP & 0.837 & 0.7 & 0.751 \\
\hline Covid-19 & 0.774 & 0.791 & 0.738 \\
\hline Accuracy & 0.71 & & \\
\hline \multicolumn{4}{|l|}{ CovidCTNet } \\
\hline Control & 0.6 & 0.75 & 0.67 \\
\hline CAP & 0.9 & 0.9 & 0.9 \\
\hline Covid-19 & 1 & 0.83 & 0.91 \\
\hline Accuracy & 0.85 & & \\
\hline
\end{tabular}

CovidCTNet can be operated as a reliable source of diagnosis. To our knowledge, despite other promising efforts ${ }^{25,29,39}$, (summarized in Table 4) CovidCTNet is an open-source framework that allows researchers and developers to adjust and build other applications based on it in a fraction of time. Besides, our approach follows the guideline proposed by Mongan et al. $^{40}$ for developing an Al method for medical image analysis.

In future efforts, we intend to (i) increase other samples as the CT scans using in this study are mostly from Iranian patients, (ii) include other demographic details of patients including age, gender and medical history to develop a predictive model, (iii) testing the model with a larger number of CT scan databases to further validate and broaden the application of our strategy.

\section{METHODS}

\section{Pre-processing}

In our effort to address the clinical diagnostic needs, with the written informed consent of patients, under institutional review board (IRB) approval (IR.TUMS.VCR.REC.1399.007, Tehran University of Medical 


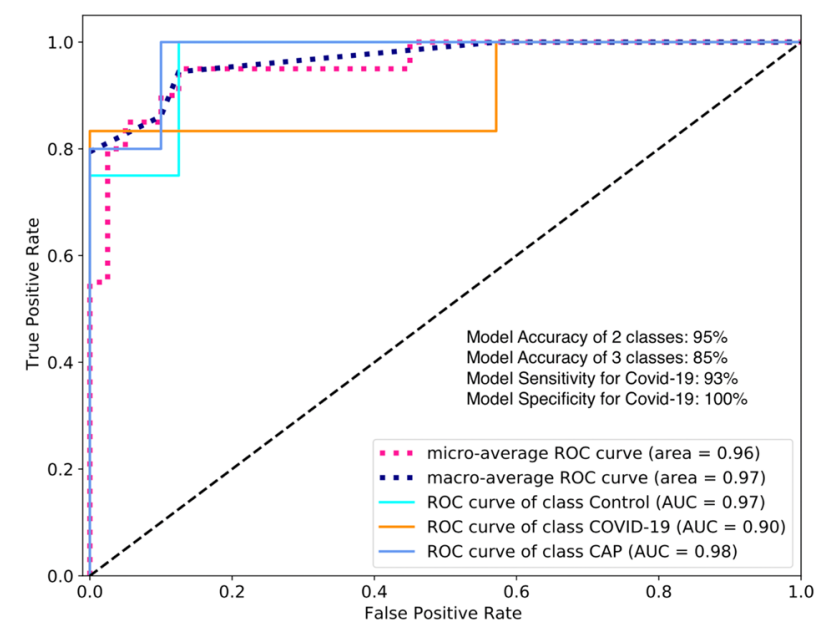

Fig. 5 Comparison of the outcome of CovidCTNet versus reader study. Performance of model and radiologists (reader) in a pool of chest CT dataset mixed of control, Covid-19 and CAP. AUC of Covid19 is 0.90 ( $n=20$ cases). The accuracy, sensitivity, and specificity of readers versus model are shown. The model operation in three classes demonstrates the detection of all three classes including Covid-19, CAP, and control separately and in two classes indicates the detection of Covid-19 as one class versus CAP and control as second class. While macroaverage takes the metric of each class independently and computes their average, the microaverage computes the average metric after aggregating the contributions of all classes.

Sciences), we collected CT images. The CT images covered a variety of image sizes, slice thicknesses, and different configurations of a range of CT scanning devices. Depending on the device and the radiologist decision, the number of scans (e.g., 60,70 , etc.), the image resolution (e.g., $512 \times 512$ pixels, $768 \times 768$ pixels, etc.), and pixel spaces in the CT images varied. Together these factors allowed us to generate a heterogeneous collection that accounts for differences in CT imaging that exist among the medical community. This broad heterogeneity within the image collection aimed to resolve the potential bias in image analysis towards a specific image quality or types of CT imaging device ${ }^{40,41}$.

In the first step of pre-processing, the $\mathrm{CT}$ slices were resampled along three axes $(z, y, x)$ to account for the variety of voxel dimensions among the CT slices (voxel is a single pixel, but in three dimensions). We used the distances of $1 \times 1 \times 1 \mathrm{~mm}$ for all voxel dimensions. Our method unified CT scans into the same scale and created a resampled dataset from the original dataset, known as resampling to an isomorphic resolution ${ }^{42}$ (https://www.kaggle.com/kmader/finding-lungs-in-ct-data) (Fig. 8 upper part). In the second pre-processing step, pixel value of the resampled CT images (3D) was optimized to have a proper range of Hounsfield Units $(\mathrm{HU})$. In our dataset, the least dense object such as air takes a value of -1000 . Lung is an organ filled with air and thus acquires a $\mathrm{HU}$ value of -700 to -600 . Other organs that may interfere with our analysis include water ( $\mathrm{HU}$ of 0 ), fat ( $\mathrm{HU}$ of -90 to -120 ) soft tissue ( $\mathrm{HU}$ of 100-300), and bone (HU of 300-1900).

Consequently, we filtered CT slices (2D) to remove non-lung tissue (e.g., skin, bone, or scanner bed) that may negatively impact our analysis and to keep only the lung related parts with an $\mathrm{HU}$ value ranging from -1000 to 400. Next, a min-max normalization is applied to rescale the -1000 and 400 numerical ranges of pixels to a 0.0 and 1.0 scale (Fig. $7 \mathrm{~b}$ ). In the third step of pre-processing, all CT slices of various pixel sizes were resized to a uniform $128 \times 128$ pixels on their $x$ and $y$ dimensions but the number of slices $(z)$ remained intact (Fig. 7b).

\section{Algorithms}

The architecture of CovidCTNet is presented in detail in Fig. 8. To identify Covid-19 in the lung from CAP lesion, we generated pseudo-infection anomalies in the CT Control images using BCDU-Net ${ }^{30}$. The BCDU-Net module played a critical role in allowing the detection of infections that have numerous features in a small dataset. It helped us to increase the accuracy and the rate of model convergence by using the initialization of the model that is trained on the Kaggle dataset for lung segmentation (https://www.kaggle.com/kmader/finding-lungs-in-ct-data). The BCDU-Net was used in our model for two purposes, first cleaning images, by removing tissues that are not related to lung infection, such as heart, skin, or the bed of CT image device, and second canceling the noise, which is used by our model for lung infection identification. To cancel the noise, $B C D U-N e t$ focused on lung infection by generating Perlin noise ${ }^{37}$ (pseudoinfection) and detecting infections. A subset of Control images mixed of noisy and non-noisy were given to BCDU-Net as an input. At the same time, the original Control images of noisy or non-noisy subsets were targeted in the model as output, mimicking the Covid-19 and CAP anomalies in the Control cases (Fig. 8 upper part). The motivation of using artificial noise (to train the model for infection detection) was to simulate both the healthy and infected state of the same lung. Therefore, the BCDUNet will learn the differences and how to extract infection from the CT images. To learn how to clean the $C T$ images, BCDU-Net received original $\mathrm{CT}$ images without noise along with the images that have noise applied on them. Afterward, the model learnt to identify and tried removing unnecessary image contents such as heart tissue. By feeding the BCDUNet with noisy CT images, the model learnt to identify infections or lesions.

To this end, we specified the input which is a combination of original healthy $\mathrm{CT}$ images and the $\mathrm{CT}$ images with noise. We defined the target of our model to be the same input CT images but without noise. Consequently, the output of BCDU-Net is de-noised and reconstructed $\mathrm{CT}$ images from the original and noisy $\mathrm{CT}$ images. Reconstructing the $\mathrm{CT}$ images helped the model to learn the pattern of the control lung and reconstruct the original Control image as output by noise reduction (denoised) and infection removal (Fig. 8b, c). Thus, Covid-19 or CAP images could not be reconstructed correctly at this stage. Identifying the Control lung pattern led to recognition of non-control slices such as Covid-19 or CAP. In the first step, the training phase (Fig. 8 upper part) starts by randomly selecting a dataset of 66 control patients (21,888 slices) and applying the pre-processing steps on their CT images (Fig. 8a, b). The dataset was divided into two subsets: (i) the original CT images of 10,944 slices (Fig. 8c right), and (ii) CT images of 10,944 slices with applied Perlin noise ${ }^{37}$ (Fig. 8c left). The BCDU-Net model was trained with two noisy and non-noisy subsets of Control images and the trained model was frozen at this step (Fig. 8d).

Next, we applied pre-processing to the entire dataset. To ensure fair comparison, the images that were used in the pre-processing step (Fig. 8 upper part), were excluded from the validation step.

Afterwards these CT slices, including Control, CAP and Covid-19 were resized (Fig. 8 middle part $e, f$ ) and fed into the frozen BCDU-Net model (Fig. 8, middle part g). The output of the BCDU-Net is the de-noised CT slices (Fig. 8, middle part h). The algorithm subtracted the output of BCDUNet, the lung slices without infection, (Fig. 8, middle part i) from the preprocessed CT slices, infected lung with Covid-19 or CAP (Fig. 8, middle part $e, f$ ) to acquire the infected areas of lung (Fig. 8, middle part i). Because the outcome of subtraction (Fig. 8, middle part i) depicted the highlighted infection area (Covid-19 and CAP) without other tissues or artifacts (Fig. 8, middle part image in violet color and Fig. 3b), it provided a reliable source for the infection classification as Covid-19 or CAP. Further examples of the result of this step are shown in Fig. 2, which shows exactly how Covid-19 and CAP infections were extracted by BCDU-Net. In other words, an example of the subtracted data (violet CT slices resulted from Fig. 8, middle part k) depicts the infection area in the lung (Fig. 3b).

In the validation, we observed that the subtraction resulted from original non-infected CT slices versus the output of BCDU-Net was insignificant, confirming the accuracy of detecting noise as an indication for the infected area. The slices at $z$-axis were concatenated to generate a 3D CT image that was the input of a three-dimensional convolutional neural network (CNN) model (Fig. 8, middle part j). The outcome of CT slices was resized due to high variation among the number of CT slices for each patient (Fig. 8, middle part k). Resizing ensures that all $C T$ images have equal sizes, which is required by CNN to have a unified size $(50 \times 128 \times 128)$. Here, 50 in the $z$ axis indicates that all the patients' CT slices were resized to 50 slices. These 3D images were already labeled by radiologists as Covid-19, CAP, or control. To implement the classification algorithm, we used CNN. In the final step, the result of Fig. 8 middle part $\mathrm{k}$ was fed into the CNN model (Fig. 8, lower part I) as a training dataset. In the training phase, the model learned to distinguish Covid-19, CAP, and control. CNN model was then validated by using 15 cases that were selected randomly and were never used before in any of the training and preceding steps. The output of the 

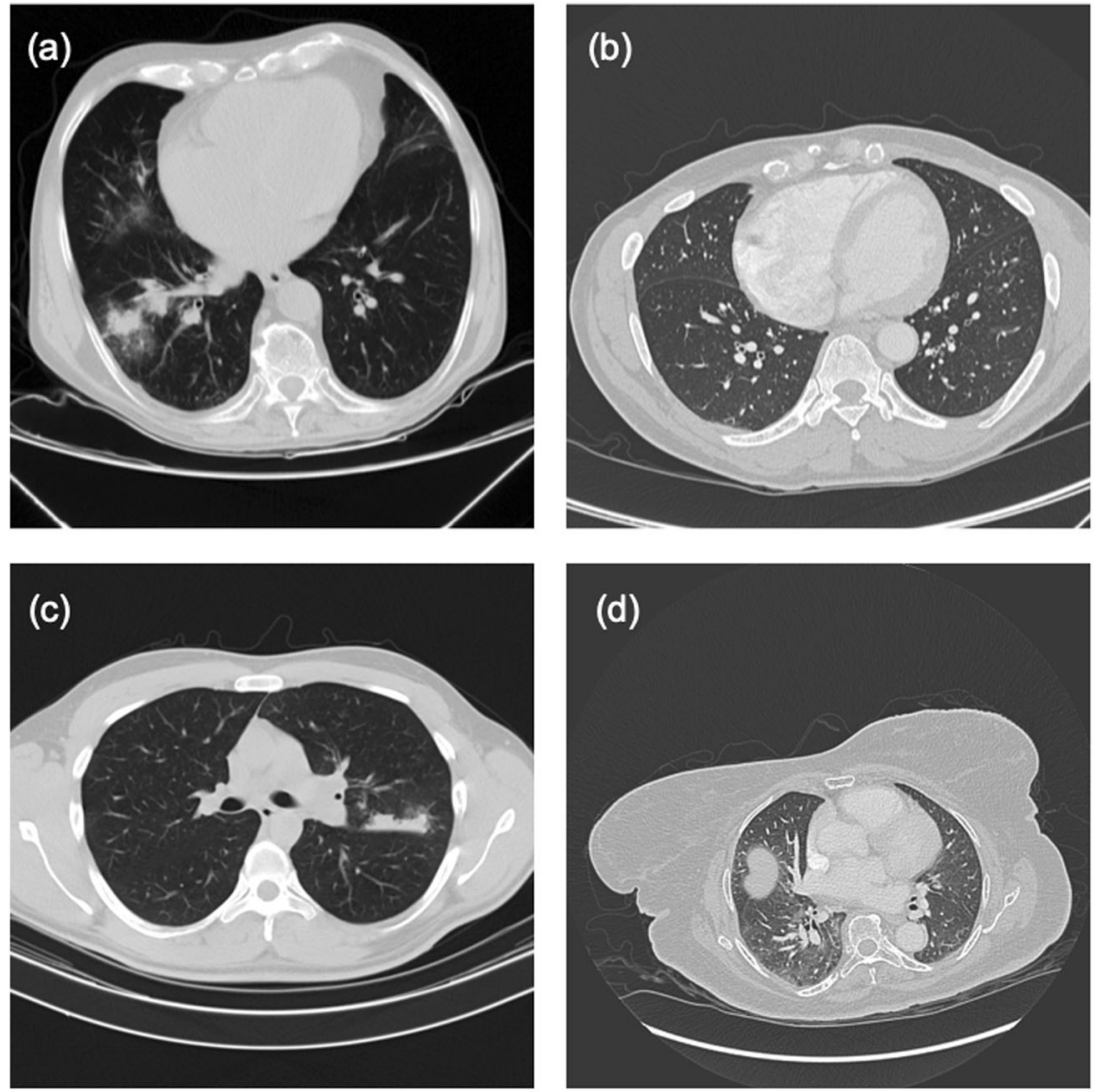

Fig. 6 Representative examples of CT images used to test the performance of CovidCTNet versus radiologists. a A CT image of CAP. This image is misidentified as Covid-19 or control by two out of four radiologists and correctly diagnosed by CovidCTNet. $\mathbf{b}$ A CT image of control, that was misdiagnosed by three out of four radiologists as Covid-19 or CAP and correctly diagnosed by CovidCTNet as control. c A sample of Covid-19 that was detected as Control by CovidCTNet and as Control or CAP by three out of the entire panel of radiologists (four members). $\mathbf{d}$ Image of control that was misdiagnosed by the CovidCTNet as CAP and by two radiologists as Covid-19 or control. Note that, in this figure one single slide of the entire scan is shown as a representative of all CT images of a patient.

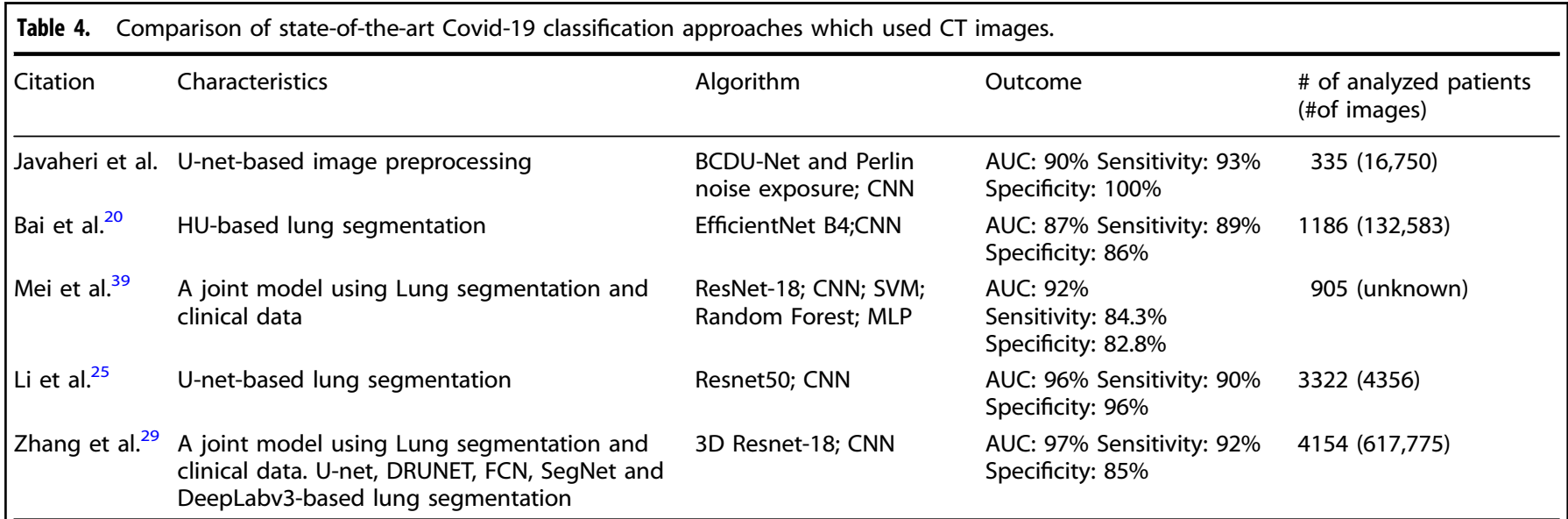

This table provides a summary on existing models including their method, achieved AUC, sensitivity, and specificity. We report here the list of approaches that rely on chest CT scans. The X-ray images were excluded from this list as they have been studied by Maguolo et al. ${ }^{41}$.

CNN algorithm is a numerical value that classifies the given patient CT images as Covid-19 or CAP or control (Fig. 8, lower part I).

The slices at $z$-axis were concatenated to generate a 3D CT image that was the input of a three-dimensional convolutional neural network (CNN) model (Fig. 8, middle part j). The outcome of CT slices was resized due to high variation among the number of $\mathrm{CT}$ slices for each patient (Fig. 8, middle part k). Resizing ensures that all CT images have equal sizes, which is required by CNN to have a unified size $(50 \times 128 \times 128)$. Here, 50 in the $z$-axis indicates that all the patients' CT slices were resized to 50 slices. These 3D images were already labeled by radiologists as Covid-19, CAP, or control. 
(a)

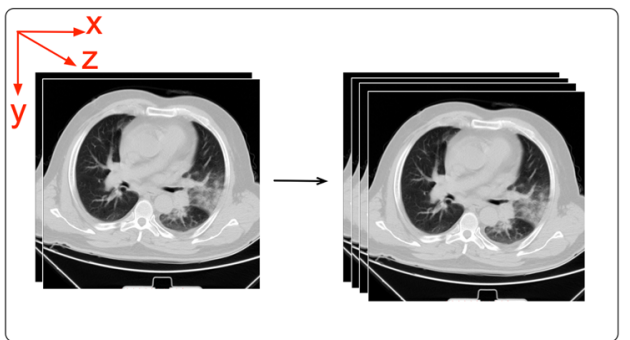

(b)

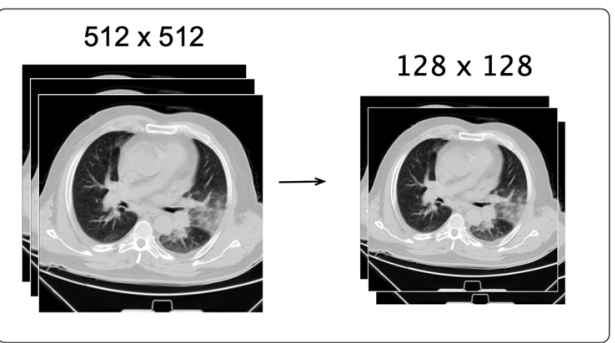

Fig. 7 Schematic representation of the pre-processing phases. a Each patient's CT image (3D) was resampled to isomorphic resolution, while $x$ and $y$ are the image coordinates and $z$ represents the number of slices. $\mathbf{b}$ All CT slices (2D) with different sizes were resized to have $128 \times 128$ pixels on the $x$ and $y$ axis, but the $z$ axis that depicts the number of slices remained intact. Here, a $512 \times 512$ pixels CT slice is resized into a $128 \times 128$ pixels CT slice.

(c)

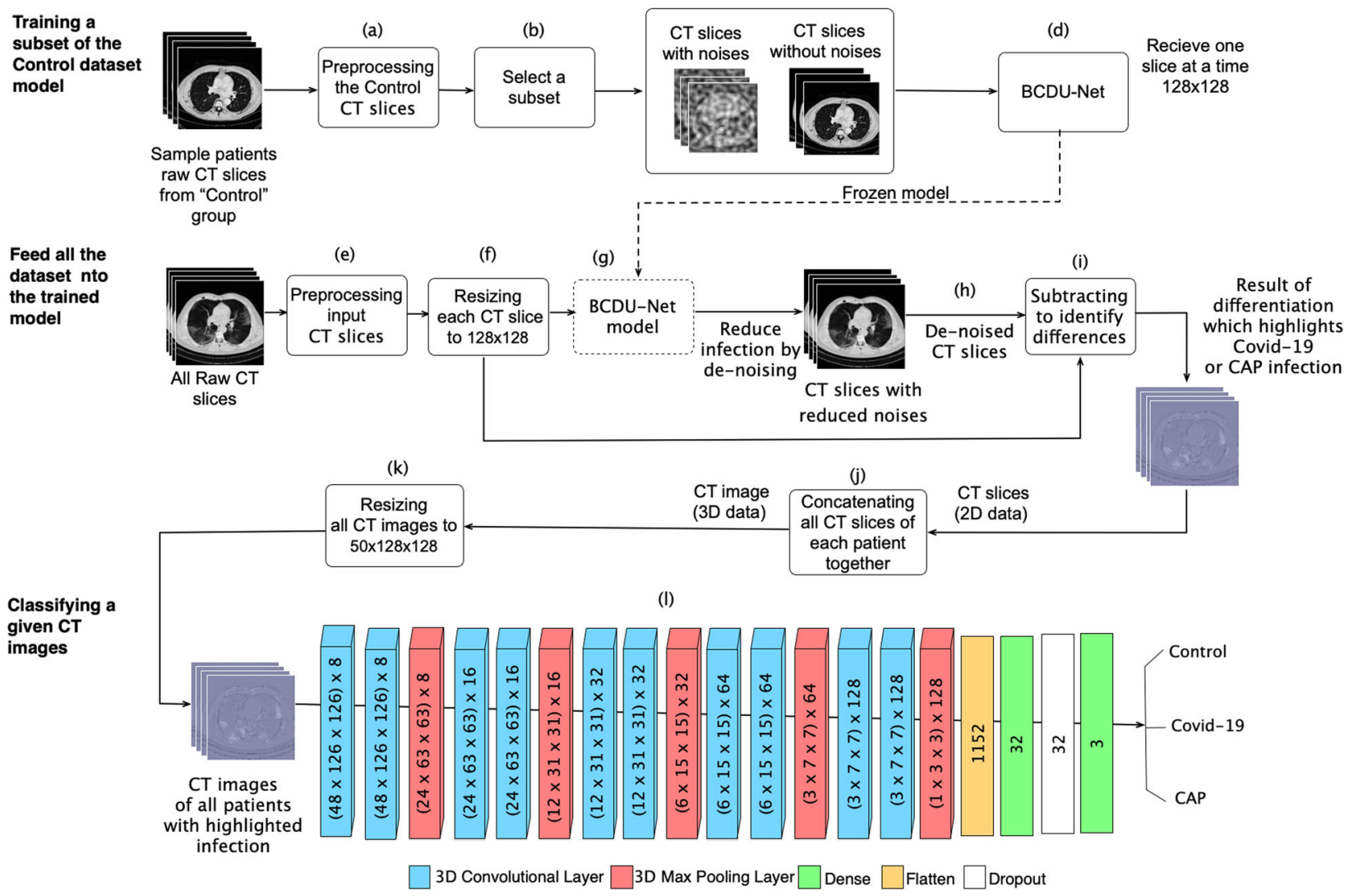

Fig. 8 Multistep pipeline of deep learning algorithms to detect Covid-19 from CT images. Upper part, Training step of the model for learning the structure of Control CT slices. Middle part, Images subtracting and lung reconstructing from CT slices with highlighted Covid-19 or CAP infection (violet color). The results of step " $\mathrm{i}$ " are a 2D image. The slices at $z$ axis concatenate to generate 3D CT image, the input of CNN model. Lower part, CNN model classifies the images that were constructed in the previous stage. To integrate this pipeline into an application the user needs to start from stage (middle part) and then the CNN algorithm recognizes whether the given CT images of a given patient presents Covid-19, CAP, or control. The number outside the parentheses in CNN model, present the number of channels in the CNN model.

\section{Reporting summary}

Further information on research design is available in the Nature Research Reporting Summary linked to this article.

\section{DATA AVAILABILITY}

The raw image dataset generated or analyzed during this study is not publicly available due to the patient privacy/consent. Datasets are available to qualified researchers following completion of a Dataset License Agreement, which is available from the corresponding author'.

\section{CODE AVAILABILITY}

To allow full reproducibility of our claims ${ }^{43,44}$ all codes are available at https:// github.com/mohofar/covidctnet. The repository contains all necessary information of model instruction and code execution. For model validation, some CT images of all three groups (Covid-19, CAP, and control) are included in the dataset.

Received: 12 May 2020; Accepted: 10 December 2020;

Published online: 18 February 2021 


\section{REFERENCES}

1. Fraser, C. et al. Pandemic potential of a strain of influenza A (H1N1): early findings. Science 324, 1557-1561 (2009).

2. WHO Coronavirus disease (COVID-19) pandemic https://www.who.int/ emergencies/diseases/novel-coronavirus-2019 Accessed 15 Aug 2020.

3. Kobayashi, T. et al. Communicating the risk of death from novel coronavirus disease (COVID-19). J. Clin. Med. 9, 580 (2020).

4. Riou, J. \& Althaus, C. L. Pattern of early human-to-human transmission of Wuhan 2019 novel coronavirus (2019-nCoV), December 2019 to January 2020. Eurosurveillance 25, 2000058 (2020).

5. Park, M., Thwaites, R. S. \& Openshaw, P. J. M. COVID-19: lessons from SARS and MERS. Eur. J. Immunol. https://doi.org/10.1002/eji.202070035 (2020).

6. Zhong, N. S. et al. Epidemiology and cause of severe acute respiratory syndrome (SARS) in Guangdong, People's Republic of China, in February 2003. Lancet 362, 1353-1358 (2003).

7. Hui, D. S. \& Chan, P. K. Severe acute respiratory syndrome and coronavirus. Infectious disease clinics of North America. Infect. Dis. Clin. 24, 619-638 (2010).

8. Fang, Y. et al. Sensitivity of chest CT for COVID-19: comparison to RT-PCR. Radiology https://doi.org/10.1148/radiol.2020200432 (2020)

9. Ai, T. et al. Correlation of chest CT and RT-PCR testing in coronavirus disease 2019 (COVID-19) in China: a report of 1014 cases. Radiology https://doi.org/10.1148/ radiol.2020200642 (2020).

10. Bernheim, A. et al. Chest CT findings in coronavirus disease-19 (COVID-19): relationship to duration of infection. Radiology https://doi.org/10.1148/ radiol.2020200463 (2020)

11. Yuan, M., Yin, W., Tao, Z., Tan, W. \& Hu, Y. Association of radiologic findings with mortality of patients infected with 2019 novel coronavirus in Wuhan, China. PLoS ONE 15, e0230548 (2020).

12. $\mathrm{Li}, \mathrm{K}$. et al. CT image visual quantitative evaluation and clinical classification of coronavirus disease (COVID-19). Eur. Radiol. https://doi.org/10.1007/s00330-02006817-6 (2020)

13. Xie, X. et al. Chest CT for typical 2019-nCoV pneumonia: relationship to negative RT-PCR testing. Radiology https://doi.org/10.1148/radiol.2020200343 (2020).

14. Spellberg, B. et al. Community prevalence of SARS-CoV-2 among patients with influenzalike illnesses presenting to a Los Angeles Medical Center in March 2020. JAMA https://doi.org/10.1001/jama.2020.4958 (2020).

15. Yan, G. et al. Covert COVID-19 and false-positive dengue serology in Singapore. Lancet Infect. Dis. https://doi.org/10.1016/S1473-3099(20)30158-4 (2020).

16. Yang, R. et al. Chest CT severity score: an imaging tool for assessing severe COVID-19. Radiology 2, e200047 (2020).

17. Wang, $\mathrm{S}$. et al. A deep learning algorithm using $\mathrm{CT}$ images to screen for corona virus disease (COVID-19). medRxiv, https://doi.org/10.1101/2020.02.14.20023028 (2020).

18. Li, Y. \& Xia, L. Coronavirus disease 2019 (COVID-19): role of chest CT in diagnosis and management. Am. J. Roentgenol. https://doi.org/10.2214/AJR.20.22954 (2020).

19. Chung, M. et al. CT imaging features of 2019 novel coronavirus (2019-nCoV). Radiology 295, 202-207 (2020).

20. Bai, H. X. et al. Performance of radiologists in differentiating COVID-19 from viral pneumonia on chest CT. Radiology https://doi.org/10.1148/radiol.2020200823 (2020).

21. Bulten, W. et al. Automated deep-learning system for Gleason grading of prostate cancer using biopsies: a diagnostic study. Lancet Oncol. 21, 233-241 (2020).

22. Shen, L. et al. Deep learning to improve breast cancer detection on screening mammography. Sci. Rep. 9, 12495 (2019).

23. Yoo, S., Gujrathi, I., Haider, M. A. \& Khalvati, F. Prostate cancer detection using deep convolutional neural networks. Sci. Rep. 9, 19518 (2019).

24. Ardila, D. et al. End-to-end lung cancer screening with three-dimensional deep learning on low-dose chest computed tomography. Nat. Med. 25, 954-961 (2019).

25. Li, L. et al. Artificial intelligence distinguishes COVID-19 from community acquired pneumonia on chest CT. Radiology https://doi.org/10.1148/radiol.2020200905 (2020).

26. Apostolopoulos, I. D. \& Mpesiana, T. A. Covid-19: automatic detection from X-ray images utilizing transfer learning with convolutional neural networks. Phys. Eng. Sci. Med. https://doi.org/10.1007/s13246-020-00865-4 (2020).

27. Sun, C., Shrivastava, A., Singh, S. \& Gupta, A. Revisiting unreasonable effectiveness of data in deep learning era. 2007 IEEE International Conference on Computer Vision (ICCV), 843-852, Venice, (2017). https://doi.org/10.1109/ICCV.2017.97.

28. Erickson, B. J. et al. Deep learning in radiology: does one size fit all? J. Am. Coll. Radiol. 15, 521-526 (2018).

29. Zhang, K. et al. Clinically applicable Al system for accurate diagnosis, quantitative measurements and prognosis of COVID-19 pneumonia using computed tomography. Cell https://doi.org/10.1016/j.cell.2020.04.045 (2020).
30. Azad, R., Asadi-Aghbolaghi, M., Fathy, M., \& Escalera, S. Bi-directional ConvLSTM U-Net with densley connected convolutions. https://arxiv.org/abs/1909.00166 (2019).

31. Linda, W. \& Alexander, W. COVID-Net: a tailored deep convolutional neural network design for detection of COVID-19 cases from chest X-ray images. https:// arxiv.org/2003.0987 (2020).

32. Chassagnon, G. et al. Al-driven CT-based quantification, staging and short-term outcome prediction of COVID-19 pneumonia. medRxiv https://doi.org/10.1101/ 2020.04.17.20069187 (2020).

33. Guan, S., Khan, A., Sikdar, S. \& Chitnis, P. Fully dense UNet for 2D sparse photoacoustic tomography artifact removal. IEEE J. Biomed. Health https://doi.org/ 10.1109/JBHI.2019.2912935 (2019).

34. Ronneberger, O., Fischer, P. \& Brox, T. Medical Image Computing and ComputerAssisted Intervention-MICCAI 2015. 234-241 (Springer, 2015).

35. Armato, S. G. III et al. SPIE-AAPM-NCI lung nodule classification challenge dataset. Cancer Imaging Arch. 10, p.K9 (2015).

36. Kirby, J. S. et al. LUNGx Challenge for computerized lung nodule classification. J. Med. Imaging 3, 044506 (2016).

37. Ken, P. An image synthesizer. Comput. Graph. 19, 287-296 (1985).

38. Hou, L. et al. Patch-based convolutional neural network for whole slide tissue image classification. In Proc IEEE Computer Society Conference on Computer Vision and Pattern Recognition, 2424-2433 (2016) https://doi.org/10.1109/CVPR.2016.266.

39. Mei, X. et al. Artificial intelligence-enabled rapid diagnosis of patients with COVID-19. Nat. Med. https://doi.org/10.1038/s41591-020-0931-3 (2020).

40. Mongan, J. et al. Checklist for artificial intelligence in medical imaging (CLAIM): a guide for authors and reviewers. Radiology https://doi.org/10.1148/ryai.2020200029 (2020).

41. Maguolo, G. et al. A critic evaluation of methods for COVID-19 automatic detection from X-ray images. https://arxiv.org/2004.12823 (2020).

42. Xie, H., Yang, D., Sun, N., Chen, Z. \& Zhang, Y. Automated pulmonary nodule detection in $\mathrm{CT}$ images using deep convolutional neural networks. Pattern Recognit. 85, 109-119 (2019).

43. Sim, I. et al. Time for NIH to lead on data sharing. Science 367, 1308-1309 (2020).

44. Benjamin, H. K. et al. The importance of transparency and reproducibility in artificial intelligence research. https://arxiv.org/2003.00898 (2020).

\section{ACKNOWLEDGEMENTS}

This research received no specific grant from any funding agency and all authors contributed solely as volunteers. We acknowledge the assistance of Prof. Reza Yousefi-Nooraei from University of Rochester, U.S. and Dr. Bita Mesgarpour from National Institute for Medical Research Development, Iran for establishing the connections between scientists from different places in the world in a very short time. Besides, we warmly appreciate Dr. Ali Babaei Jandaghi from Princess Margaret Cancer Centre, Canada and Dr. Nahid Sadighi from Tehran University of Medical Sciences, Iran for sharing their expertize on CT images annotation. We dedicate our results to all nurses and physicians around the world.

\section{AUTHOR CONTRIBUTIONS}

R.R., (Rawassizadeh) planned and directed the study. T.J. and R.R. (Rawassizadeh) conceived and planned the study. M.H. and R.R., (Rawassizadeh) designed the model and the computational framework and analysed the data. T.J. and Z.A. took the lead in writing the manuscript and contributed to the interpretation of the results. R.R. (Reiazi) performed the data analysis and calculations. F.H., E.A., A.L., A.R.R., M.H.G., S.A J.M., O.G., R.B., H.K.M., M.H., R.J., K.S., M.K.K., G.Z., L.T.C., B.H.K., and R.M. contributed to image collection, provided critical feedback and helped to shape the research, analysis and manuscript.

\section{COMPETING INTERESTS}

The authors declare no competing interests.

\section{ADDITIONAL INFORMATION}

Supplementary information The online version contains supplementary material available at https://doi.org/10.1038/s41746-021-00399-3.

Correspondence and requests for materials should be addressed to R.R.

Reprints and permission information is available at http://www.nature.com/ reprints 
Publisher's note Springer Nature remains neutral with regard to jurisdictional claims in published maps and institutional affiliations.

(c) Open Access This article is licensed under a Creative Commons Attribution 4.0 International License, which permits use, sharing, adaptation, distribution and reproduction in any medium or format, as long as you give appropriate credit to the original author(s) and the source, provide a link to the Creative
Commons license, and indicate if changes were made. The images or other third party material in this article are included in the article's Creative Commons license, unless indicated otherwise in a credit line to the material. If material is not included in the article's Creative Commons license and your intended use is not permitted by statutory regulation or exceeds the permitted use, you will need to obtain permission directly from the copyright holder. To view a copy of this license, visit http://creativecommons. org/licenses/by/4.0/.

(c) The Author(s) 2021 